PROCEEDINGS OF THE

AMERICAN MATHEMATICAL SOCIETY

Volume 137, Number 4, April 2009, Pages 1303-1305

S 0002-9939(08)09737-2

Article electronically published on October 28, 2008

\title{
TRANSCENDENCE OF POWER SERIES FOR SOME NUMBER THEORETIC FUNCTIONS
}

\author{
PETER BORWEIN AND MICHAEL COONS
}

(Communicated by Ken Ono)

\begin{abstract}
We give a new proof of Fatou's theorem: if an algebraic function has a power series expansion with bounded integer coefficients, then it must be a rational function. This result is applied to show that for any non-trivial completely multiplicative function from $\mathbb{N}$ to $\{-1,1\}$, the series $\sum_{n=1}^{\infty} f(n) z^{n}$ is transcendental over $\mathbb{Z}(z)$; in particular, $\sum_{n=1}^{\infty} \lambda(n) z^{n}$ is transcendental, where $\lambda$ is Liouville's function. The transcendence of $\sum_{n=1}^{\infty} \mu(n) z^{n}$ is also proved.
\end{abstract}

In 1945 Duffin and Schaeffer 8 proved that a power series that is bounded in a sector and has coefficients from a finite subset is already a rational function. Their proof is relatively indirect. In [5], the first author, Erdélyi, and Littmann gave a shorter direct proof of this beautiful and surprising theorem.

The theorem of Duffin and Schaeffer is a generalization of a result of Szegö, who proved in 1922 that a power series $f$ whose coefficients assume only finitely many values and which can be extended analytically beyond the unit circle is already a rational function.

In 1906 Fatou [10] proved, and in 1999 Allouche [1] re-proved using a deep result of Cobham [6], that

Theorem 1 (Fatou, 1906). A power series whose coefficients take only finitely many values is either rational or transcendental.

In this paper, we give a new proof of Fatou's theorem and apply it to show that various power series are transcendental; as specific examples we show the transcendence of the series $\sum_{n=1}^{\infty} \lambda(n) z^{n}$ and $\sum_{n=1}^{\infty} \mu(n) z^{n}$, where $\lambda$ and $\mu$ are the Liouville and Möbius functions, respectively.

We will need the following quantitative version of the Fundamental Theorem of Algebra, Theorem 1.2.1 of [4].

Lemma 1. The polynomial

$$
p(z):=a_{n} z^{n}+a_{n-1} z^{n-1}+\cdots+a_{0}, \quad a_{n} \neq 0,
$$

has exactly $n$ zeros. These all lie in the open disk of radius $r$ centered at the origin, where

$$
r:=1+\max _{0 \leq k \leq n-1} \frac{\left|a_{k}\right|}{\left|a_{n}\right|} .
$$

Received by the editors May 30, 2008.

2000 Mathematics Subject Classification. Primary 11J81, 11J99; Secondary 30B10, 26C15.

Research supported in part by grants from NSERC of Canada and MITACS.

(C) 2008 By the authors 
Proof of Theorem 1. Suppose that $f(z)$ satisfies

$$
a_{n}(z) f(z)^{n}+a_{n-1}(z) f(z)^{n-1}+\cdots+a_{0}(z)=0,
$$

where each $a_{i}(z)$ is a polynomial with integer coefficients. Since the leading coefficient $a_{n}(z)$ of this polynomial equation is a polynomial, it has finitely many zeros. Hence there is a sector $S$ of the open unit disk where $\left|a_{n}(z)\right|$ is bounded away from zero uniformly. The modulus of each other coefficient $a_{k}(z)$ is clearly uniformly bounded above on $S$. Now apply Lemma 1 to conclude that $|f(z)|$ is bounded on $S$, so the result of Duffin and Schaeffer applies.

Denote by $\mu$ the Möbius function and by $\lambda$ the Liouville function. Recall that $\lambda$ is the unique completely multiplicative function defined by $\lambda(p)=-1$ for all primes $p$.

In 2 it is shown that the formal power series $\sum_{n=1}^{\infty} \lambda(n) z^{n}, \sum_{n=1}^{\infty} \mu(n) z^{n} \in$ $\mathbb{Z}[[z]]$ are irrational over $\mathbb{Z}(z)$ (and various other multiplicative functions). We proceed by proving that these two power series are transcendental over $\mathbb{Z}(z)$. The transcendence of $\sum_{n=1}^{\infty} \lambda(n) z^{n}$ is stated as a corollary to the following general theorem.

Theorem 2. Let $f: \mathbb{N} \rightarrow\{-1,1\}$ be a completely multiplicative function with the property that for some prime $p, f(p)=-1$. Then $\sum_{n=1}^{\infty} f(n) z^{n} \in \mathbb{Z}[[z]]$ is transcendental over $\mathbb{Z}(z)$.

Proof. In light of Theorem 1, we need only demonstrate that for a completely multiplicative function $f: \mathbb{N} \rightarrow\{-1,1\}$ such that there is a prime $p$ for which $f(p)=-1$, the sequence of values of $f$ is not eventually periodic. This would show that $\sum_{n=1}^{\infty} f(n) 2^{-n}$ is irrational. Denote the sequence of values of $f$ by $\mathfrak{F}$.

Towards a contradiction, suppose that $\mathfrak{F}$ is eventually periodic, say the sequence is periodic after the $M$-th term and has period $k$. Now there is an $N \in \mathbb{N}$ such that for all $n \geq N$, we have $n k>M$. Let $p$ be a prime for which $f(p)=-1$. Then

$$
f(p n k)=f(p) f(n k)=-f(n k) .
$$

But $p n k \equiv n k(\bmod k)$, a contradiction to the eventual $k$-periodicity of $\mathfrak{F}$.

Corollary 1. If $\lambda$ is the Liouville function, then the series $\sum_{n=1}^{\infty} \lambda(n) z^{n} \in \mathbb{Z}[[z]]$ is transcendental over $\mathbb{Z}(z)$.

Note that Theorem 2 does not apply directly to the Möbius function; $\mu$ is not completely multiplicative. Recall from the definition that if $p^{2} \mid n$ for any prime $p$, then $\mu(n)=0$. From this fact alone one may use the Chinese Remainder Theorem to show that the sequence of values of the Möbius function contains arbitrarily long runs of zeroes. This in turn gives the irrationality of $\sum_{n=1}^{\infty} \mu(n) z^{n}$ at $z=\frac{1}{3}$ and hence the following corollary to Theorem [1.

Corollary 2. If $\mu$ is the Möbius function, then the series $\sum_{n=1}^{\infty} \mu(n) z^{n} \in \mathbb{Z}[[z]]$ is transcendental over $\mathbb{Z}(z)$.

The transcendence of the series $\sum_{n=1}^{\infty} f(z) z^{n}$ over $\mathbb{Z}(z)$ for $f$ equal to each of the multiplicative functions $\tau_{k}, \sigma_{k}$, and $\varphi$ was shown by Yazdani [12] the case $f=\mu$ was previously treated by Allouche [1] using a deep result of Cobham [7]. While transcendence results on power series are readily available, there are many open questions concerning their special values. Erdős 9] was interested in the transcendence (and irrationality) of both $\sum_{n=1}^{\infty} \tau(n) 2^{-n}$ and $\sum_{n=1}^{\infty} \varphi(n) 2^{-n}$. The 
irrationality of the first sum was shown by Borwein [3]; the transcendence is not yet known. Questions regarding the algebraic character of $\sum_{n=1}^{\infty} \varphi(n) 2^{-n}$ remain open. Nesterenko [1] has shown that the number

$$
\sum_{n=1}^{\infty} \frac{\lambda(n)}{2^{n}-1}
$$

is transcendental, though the transcendence of its close relative, $\sum_{n=1}^{\infty} \lambda(n) 2^{-n}$, remains elusive.

\section{REFERENCES}

1. J.-P. Allouche, Transcendence of formal power series with rational coefficients, WORDS (Rouen, 1997), Theoret. Comput. Sci. 218 (1999), no. 1, 143-160. MR1687784(2000e:13029)

2. W. D. Banks, F. Luca, and I. E. Shparlinski, Irrationality of power series for various number theoretic functions, Manuscripta Math. 117 (2005), no. 2, 183-197. MR2150480|(2006c:11081)

3. Peter B. Borwein, On the irrationality of $\sum\left(1 /\left(q^{n}+r\right)\right)$, J. Number Theory 37 (1991), no. 3, 253-259. MR1096442 (92b:11046)

4. P. Borwein and T. Erdélyi, Polynomials and polynomial inequalities, Graduate Texts in Mathematics, vol. 161, Springer-Verlag, New York, 1995. MR1367960 (97e:41001)

5. P. Borwein, T. Erdélyi, and F. Littmann, Polynomials with coefficients from a finite set, Trans. Amer. Math. Soc. 360 (2008), 5145-5154.

6. A. Cobham, On the base-dependence of sets of numbers recognizable by finite automata, Math. Systems Theory 3 (1969), 186-192. MR0250789 (40:4021)

7. A. Cobham, Uniform tag sequences, Math. Systems Theory 6 (1972), 164-192. MR0457011 $(56: 15230)$

8. R. J. Duffin and A. C. Schaeffer, Power series with bounded coefficients, Amer. J. Math. 67 (1945), 141-154. MR0011322 (6:148g)

9. P. Erdös, On the irrationality of certain series: problems and results, New Advances in Transcendence Theory (Durham, 1986), Cambridge Univ. Press, Cambridge, 1988, pp. 102109. MR971997 (89k:11057)

10. P. Fatou, Séries trigonométriques et séries de Taylor, Acta Math. 30 (1906), no. 1, 335-400. MR 1555035

11. Yu. V. Nesterenko, Modular functions and transcendence questions, Mat. Sb. 187 (1996), no. 9, 65-96. MR1422383 (97m:11102)

12. S. Yazdani, Multiplicative functions and $k$-automatic sequences, J. Théor. Nombres Bordeaux 13 (2001), no. 2, 651-658. MR.1879677 (2003a:11019)

Department of Mathematics, Simon Fraser University, British Columbia, Canada V5A 1 S6

E-mail address: pborwein@cecm.sfu.ca

Department of Mathematics, Simon Fraser University, British Columbia, Canada V5A 1 S6

E-mail address: mcoons@sfu.ca 